\title{
Analytical methods for detecting pesticide switches with evolution of pesticide resistance
}

\author{
Juhua Liang ${ }^{a}$, Sanyi Tang ${ }^{\mathrm{a}, *}$, Juan J. Nieto ${ }^{\mathrm{b}}$, Robert A. Cheke ${ }^{\mathrm{c}}$ \\ ${ }^{a}$ College of Mathematics and Information Science, Shaanxi Normal University, Xi'an 710062, People's Republic of China \\ bepartamento de Análisis Matemático, Universidad de Santiago de Compostela, Santiago de Compostela 15782, Spain \\ ${ }^{\mathrm{c}}$ Natural Resources Institute, University of Greenwich at Medway, Central Avenue, Chatham Maritime, Chatham, Kent ME4 4TB, UK
}

\section{A R T I C L E I N F O}

\section{Article history:}

Received 5 November 2012

Received in revised form 14 July 2013

Accepted 15 July 2013

Available online 24 July 2013

\section{Keywords:}

Pesticide switches

Dynamic threshold

Pesticide application frequency

Evolution

Pulse spraying

\begin{abstract}
A B S T R A C T
After a pest develops resistance to a pesticide, switching between different unrelated pesticides is a common management option, but this raises the following questions: (1) What is the optimal frequency of pesticide use? (2) How do the frequencies of pesticide applications affect the evolution of pesticide resistance? (3) How can the time when the pest population reaches the economic injury level (EIL) be estimated and (4) how can the most efficient frequency of pesticide applications be determined? To address these questions, we have developed a novel pest population growth model incorporating the evolution of pesticide resistance and pulse spraying of pesticides. Moreover, three pesticide switching methods, threshold condition-guided, density-guided and EIL-guided, are modelled, to determine the best choice under different conditions with the overall aim of eradicating the pest or maintaining its population density below the EIL. Furthermore, the pest control outcomes based on those three pesticide switching methods are discussed. Our results suggest that either the density-guided or EIL-guided method is the optimal pesticide switching strategy, depending on the frequency (or period) of pesticide applications.
\end{abstract}

(C) 2013 Elsevier Inc. All rights reserved.

\section{Introduction}

Pesticide resistance is the adaptation of a pest population targeted by a pesticide, resulting in decreased susceptibility of the pest to the chemical. Pesticide resistance is increasing and farmers' and other pest managers' dependencies on chemical insecticides have led to a high frequency of insecticide resistance in some crop systems [1]. In the 1940s, farmers in the USA lost $7 \%$ of their crops to pests. Since the 1980s, the percentage lost has increased to $13 \%$, even though more pesticides are being used, this is because more than 500 species of pests have developed resistance to pesticides since 1945 [2-4], and the situation is often caused by the same classes of pesticides being used repeatedly for a long time. Other problems ensue such as pest resurgence, acute and chronic health problems, environmental pollution and uneconomic crop production.

Therefore, knowledge of the mechanisms for the evolution of pesticide resistance is important for developing strategies to avoid the creation of resistance in pest populations, with the underlying principle being the preservation of susceptible genes in pest populations. Therefore, in order to fight pesticide resistance and based on a knowledge of the genetics of the development of pesticide

\footnotetext{
* Corresponding author. Tel.: +86 15934823867.

E-mail addresses: sytang@snnu.edu.cn, sanyitang219@hotmail.com (S. Tang).
}

resistance, a number of principles have been proposed aimed at delaying the emergence of resistance or avoiding it entirely. These principles include pesticide rotation or switching, avoiding unnecessary pesticide applications, using non-chemical control techniques [5], and leaving untreated refuges where susceptible pests can survive, within the concept of integrated pest management (IPM) [6-10].

When pesticides are the sole or predominant method of pest control, resistance is commonly managed through pesticide rotations or pesticide switches. This means after a pest species develops resistance to a particular pesticide, one method is to use a different pesticide, especially one in a different chemical class or family of pesticides that has a different mode of action against the pest. So far, switching among unrelated insecticides in response to detection of resistance has been the main method used. For instance, during the WHO Onchocerciasis Control Programme $(\mathrm{OCP})$ in West Africa examples of different categories of pesticides were used in rotation after the blackfly vectors of Onchocerciasis developed resistance to the chemical of choice, the organophosphate temephos [11]. Similarly, in agriculture, insecticide rotation has been widely used to combat resistance in a major pest of brassica crops, the Diamondback Moth Plutella xylostella [12].

To achieve pest resistance management using pesticide switches or rotations, the key problems that we are facing are: What is the optimal frequency of pesticide use? How do the 
frequencies of pesticide applications affect the evolution of pesticide resistance and when does the pest population reach the critical threshold value?

In order to address those questions, mathematical models can be useful for determining the optimal frequency of pesticide applications, when is best to switch pesticides and for predicting how fast pesticide resistance develops. To do this, we have developed a novel pest population growth model concerning evolution of pest resistance and pulse spraying of pesticides. The model incorporates three different pesticide switching tactics for eradicating the pest or maintaining its population density below a given critical level.

The first justification for stopping the use of a given pesticide and switching a new type of pesticide (so called as pesticide switching method throughout this paper) is based on the threshold condition (the threshold condition-guided method) which ensures the extinction of the pest population, i.e. the pesticide is switched once the threshold value increases due to evolution of pesticide resistance and exceeds one, which determines the stability of pest eradication solutions.

The second pesticide switching method depends on the density of the pest population just before the pesticide is applied (the density-guided method). This switching action occurs when the efficacy of the pesticide begins to wear off, i.e. there is resurgence.

An important concept in IPM is that of the economic threshold (ET), which is usually defined as the number of pests in the field when control actions must be taken to prevent the economic injury level (EIL) from being reached and exceeded. The EIL is defined as the lowest pest population density that will cause economic damage [6,8-10]). For an IPM strategy, action must be taken once a critical density of pests is observed in the field so that the EIL is not exceeded. Thus, the third switching action is instigated when the pest population reaches the EIL (the EIL-guided method).

We provide analytical formulae for the optimal times to switch between different unrelated pesticides for all of the above three methods. Based on different situations, the optimal choices for each of these three methods, with the intention of eradicating the pests or maintaining their population density below a tolerable level, are discussed. Our results suggest that either the density-guided or the EIL-guided method is the optimal pesticide switching strategy, depending on the frequency (or period) of the pesticide applications.

\section{Pest growth model with evolution of pesticide resistance}

In this section, we will develop a simple pest population growth model concerning the evolution of pest resistance. In particular, the effects of the frequency of pesticide applications are modelled and investigated. One of our main purposes is to investigate how to implement a chemical control strategy and manage pest resistance such that the pest population dies out eventually or its density is maintained below the EIL. In order to address this topic, we focus on the threshold condition which guarantees the extinction of the pest population and discuss optimal strategies for pesticide switches.

\subsection{Simple pest growth model with pesticide resistance}

Throughout this study, the pest population is assumed to grow logistically with an intrinsic growth rate $r$ and a carrying capacity parameter $\eta$. Then the pest population follows

$\frac{d P}{d t}=r P(1-\eta P)$

In the following, the total pest population is divided into two parts: susceptible pests (denoted by $P_{S}$ ) and resistant pests (denoted by $P_{R}$ ), and the proportion of susceptible pests in the population is denoted by a fraction $\omega$, the remaining fraction $1-\omega$ is resistant, so we have $P_{S}=\omega P$ and $P_{R}=(1-\omega) P$. Susceptible pests are those that have not developed resistance to the pesticide. That is to say, $\omega$ may be thought of as the stock of effectiveness of the pesticide, and it is the proportion of the pest population to which the toxin is lethal. Naturally, the susceptible pests are assumed to die with a higher mortality rate, $d_{1}$, and the resistant pests are assumed to die with mortality rate, $d_{2}$, when chemical control is implemented. Then the growth of susceptible and resistant pests can be modelled as follows:

$\left\{\begin{array}{l}\frac{d P_{S}}{d t}=\omega r P(1-\eta P)-d_{1} P_{S}, \\ \frac{d P_{R}}{d t}=(1-\omega) r P(1-\eta P)-d_{2} P_{R} .\end{array}\right.$

However, for simplification we assume that the resistant pests display near-complete resistance to the pesticide, which means that $d_{2} \approx 0$ [13]. Consequently, the evolution of the total pest population follows

$\frac{d P}{d t}=\frac{d P_{S}}{d t}+\frac{d P_{R}}{d t}=r P(1-\eta P)-\omega d_{1} P$

Since $\omega=P_{S} / P$, then the evolution of the fraction of the susceptible pests in the total pest population is

$\frac{d \omega}{d t}=\frac{d}{d t}\left(\frac{P_{S}}{P}\right)=\left(\frac{d P_{S}}{d t} P-P_{S} \frac{d P}{d t}\right) / P^{2}=d_{1} \omega(\omega-1)$.

Note that this resistance evolution equation has been widely used recently in different fields [13-17].

Therefore, the model (1) can be written as

$\left\{\begin{array}{l}\frac{d P}{d t}=r P(1-\eta P)-\omega d_{1} P, \\ \frac{d \omega}{d t}=d_{1} \omega(\omega-1) .\end{array}\right.$

In reality, the pesticides are applied instantaneously. Thus the model (4) can be developed by introducing an impulsive spraying of pesticide at a critical time and modelling the consequences of population densities changing very rapidly.

If the pesticides is applied at time point $\tau_{i-1}$ for $i \in \mathcal{N}$ with $\tau_{0}=0$, where $\mathcal{N}=\{1,2,3, \ldots\}$ and $0=\tau_{0}<\tau_{1}<\tau_{2}<\cdots$, then the number of pests killed at time $\tau_{i-1}$ is $d_{1} \omega\left(\tau_{i-1}\right) P\left(\tau_{i-1}\right)$. Therefore, we have the following impulsive differential equation

$\begin{cases}\frac{d P(t)}{d t}=r P(t)(1-\eta P(t)), & t \neq \tau_{i}, \\ P\left(\tau_{i}^{+}\right)=\left(1-\omega\left(\tau_{i}\right) d_{1}\right) P\left(\tau_{i}\right), & t=\tau_{i}, \\ \frac{d \omega(t)}{d t}=d_{1} \omega(t)(\omega(t)-1), & \end{cases}$

where $P\left(\tau_{0}^{+}\right)=P_{0}$ and $\omega\left(\tau_{0}\right)=\omega_{0}$. This indicates that the initial condition of the pest population in model (5) is chosen as the population density after the first application of pesticide at time $\tau_{0}$.

It is clear from model (5) that the efficacy of the pesticide on the target pest population depends on the evolution of pest resistance, as the killing efficacy will decrease as pest resistance develops. A detailed analysis of model (5) will be given in the coming sections.

\subsection{The effects of frequency of pesticide applications on evolution of resistance}

The formula (3) indicates how the pest resistance develops with respect to time. However, it does not involve the effects of the frequency of pesticide applications, the pesticide application period or the dosage of the applications on the evolution of resistance, and those factors do influence resistance patterns. Although it is difficult to involve these factors in the model (3), we note that the speed with which resistance develops depends on several factors including the rate, timing and number of applications made. Based on this fact, we assume that at each time point 
$\tau_{i-1}, i \in \mathcal{N}$, one pulse of pesticide is applied, which yields the following equation for the fraction of susceptible pests

$\frac{d \omega(t)}{d t}=d_{1} \omega\left(\omega^{q_{i}}-1\right), \quad \tau_{i-1} \leqslant t \leqslant \tau_{i}, i \in \mathcal{N}$

with initial value $\omega\left(\tau_{i-1}\right)$ at each time interval $\tau_{i-1} \leqslant t \leqslant \tau_{i}$ and $\omega\left(\tau_{0}\right)=\omega(0)=\omega_{0}$ is given, where $q_{i}$ should be a function of the number of pesticide applications, dosage $D_{i}$ of the $i$ th pesticide application and time interval $\Delta \tau_{i}=\tau_{i}-\tau_{i-1}$ between the $i$ th and $(i-1)$ th pesticide applications. For simplification, we assume that each time the same dosage of pesticide is applied, i.e. $D_{i}$ is a constant and without loss of generality we let $D_{i}=1$ for all $i \in \mathcal{N}$. Thus the simplest formula for $q_{i}$ could be defined as $q_{i}=i / \Delta \tau_{i}$. For example, if $i=1$, then the differential equation

$\frac{d \omega(t)}{d t}=d_{1} \omega\left(\omega^{q_{1}}-1\right), \quad \tau_{0} \leqslant t \leqslant \tau_{1}, \omega(0)=\omega_{0}$

describes the evolution of the fraction of susceptible pests at time interval $\tau_{0} \leqslant t \leqslant \tau_{1}$ after the first pesticide is applied at time $\tau_{0}$. Numerical investigations clarify that the model (6) does describe the effects of the timing and number of pesticide applications on the development of resistance, as shown in Fig. 1.

In order to show how the frequency of pesticide applications (or the period of pesticide application) affects the evolution of pest resistance, we let $\Delta \tau_{i}$ be a constant $T$ (i.e. pesticide applied periodically) and show the effects of different periods on the evolution of $\omega$. In Fig. 1 four curves for $\omega(t)$ are plotted with respect to $q_{i}=i, i / 2, i / 3$ and constant 1 , from which it is clear how the frequency of pesticide applications and different $q_{i}$ functions affect the evolution of pest resistance, i.e. the higher the frequency of pesticide applications, the faster the evolution of pest resistance. For simplicity, we will focus on the periodical applications of pesticides in the rest of this work.

The analytical solution of Eq. (6) can be solved as

$\omega(t)=\left(1+e^{q_{i} d_{1}\left(t-\tau_{i-1}\right)}\left(\left(\omega\left(\tau_{i-1}\right)\right)^{-q_{i}}-1\right)\right)^{-1 / q_{i}}, \quad \tau_{i-1} \leqslant t \leqslant \tau_{i}$, which indicates that

$\omega\left(\tau_{i}\right)=\left(1+e^{i d_{1}}\left(\omega\left(\tau_{i-1}\right)^{-q_{i}}-1\right)\right)^{-1 / q_{i}}$.

If we apply pesticide periodically, i.e. $\Delta \tau_{i}=T$ for all $i \in \mathcal{N}$, then the evolution of $\omega$ at each time point $n T$ can be expressed as

$\omega(n T)=\left(1+e^{n d_{1}}\left(\omega((n-1) T)^{-n / T}-1\right)\right)^{-T / n}, \quad n \in \mathcal{N}$.

In particular, if $q_{i}=1$, i.e. the evolution of $\omega(t)$ satisfies Eq. (3), then

$\omega(t)=\frac{\omega_{0}}{\omega_{0}+\left(1-\omega_{0}\right) e^{d_{1} t}}, t \geqslant 0$.

\section{Threshold conditions and justifications and the optimal time to switch pesticides}

\subsection{Threshold condition for pest extinction}

If we take into account the effects of the frequency of pesticide applications on the evolution of pest resistance, then model (5) becomes the following periodic control model

$$
\begin{cases}\frac{d P(t)}{d t}=r P(t)(1-\eta P(t)), & t \neq n T, \\ P\left(n T^{+}\right)=\left(1-\omega(n T) d_{1}\right) P(n T), & t=n T, \\ \frac{d \omega(t)}{d t}=d_{1} \omega(t)\left(\omega(t)^{q_{n}}-1\right), & \end{cases}
$$

where $T$ is the period of pesticide application and $q_{n}=n / T, P\left(0^{+}\right)=P_{0}, \omega(0)=\omega_{0}$.

Note that the third equation of model (11) is independent of the pest population, and then $\omega(t)$ can be analytically solved which is given by (9).

Furthermore, the density of the pest population in the first equation of model (11) can be solved as

$$
P(t)=\frac{\left(1-\omega(n T) d_{1}\right) P(n T) e^{r(t-n T)}}{1+\left(1-\omega(n T) d_{1}\right) P(n T) \eta\left(e^{r(t-n T)}-1\right)}
$$

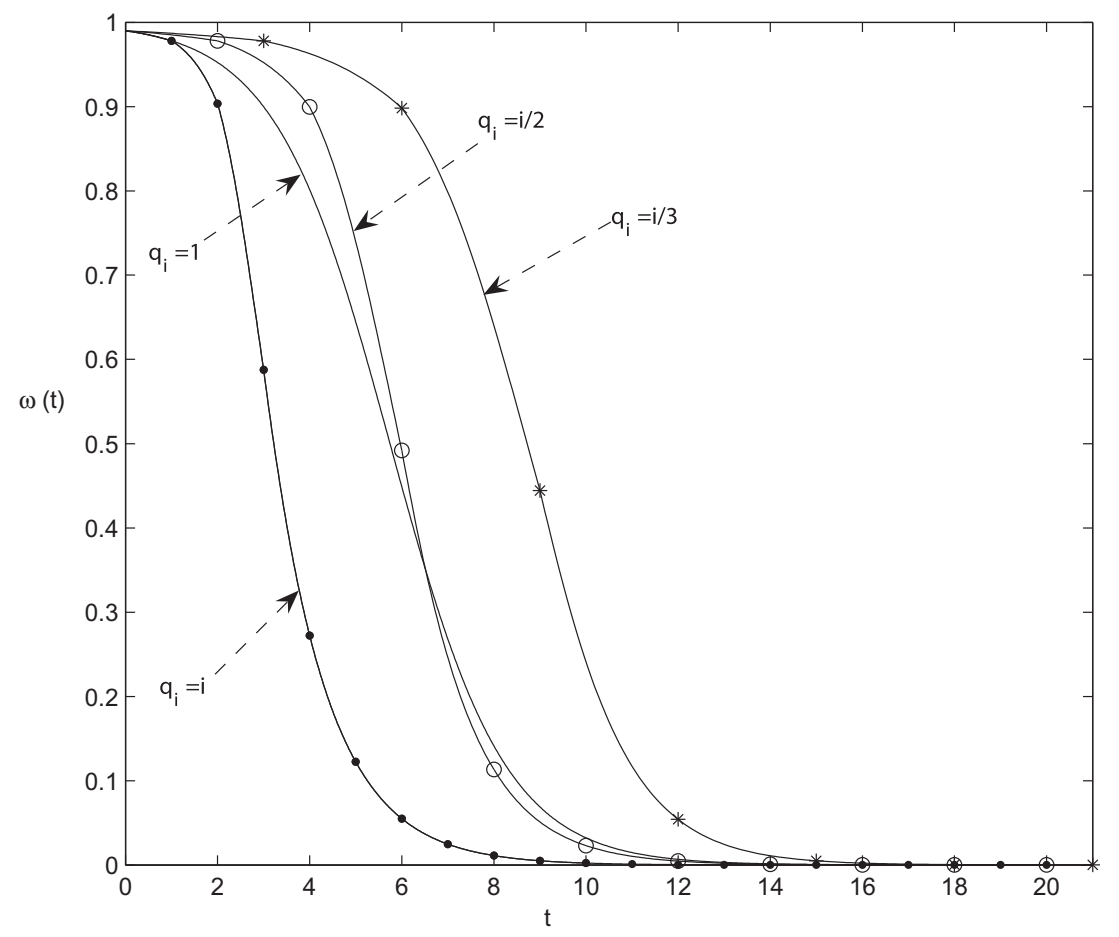

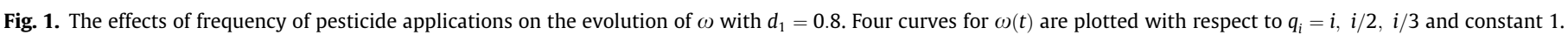


(a)

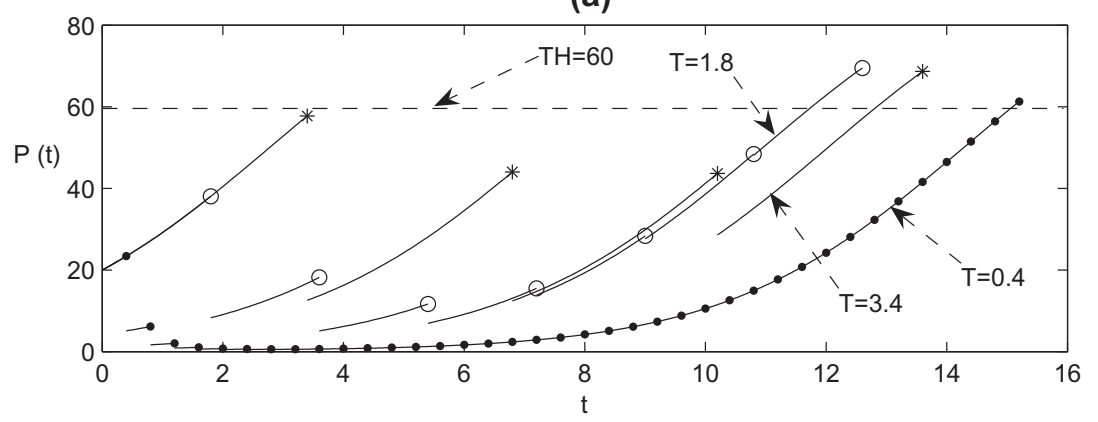

(b)

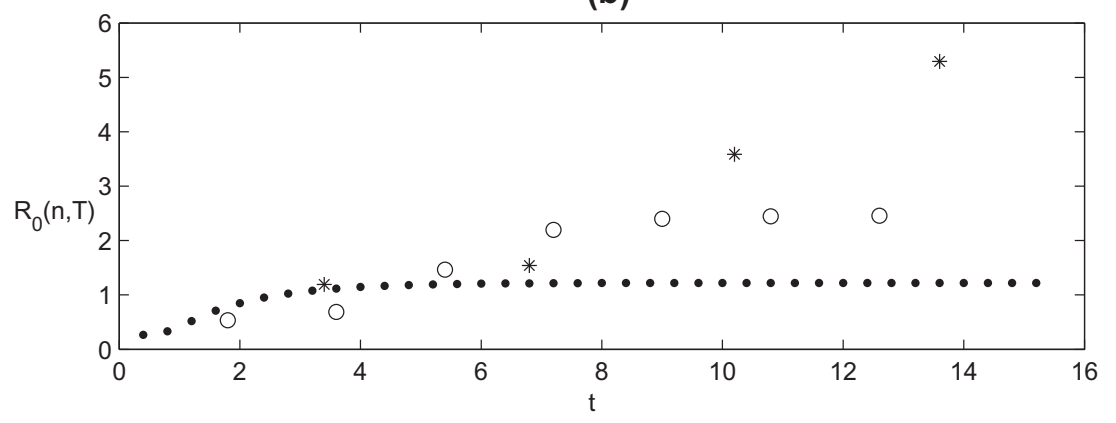

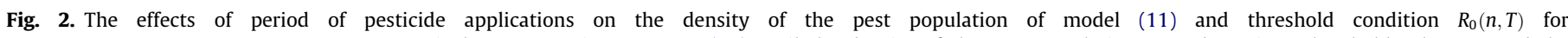

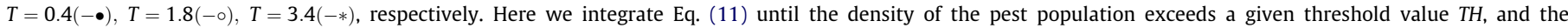

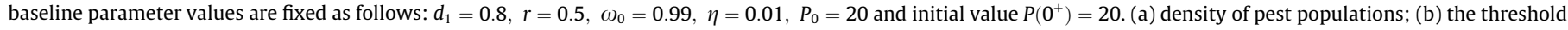

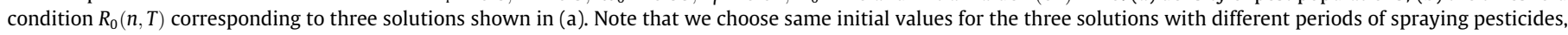
so the first times of spraying pesticide for three different periods lie on the same curve in (a).

for any pulse interval $n T<t \leqslant(n+1) T, n=0,1,2, \ldots$. Therefore,

$P((n+1) T)=\frac{\left(1-\omega(n T) d_{1}\right) e^{r T} P(n T)}{1+\left(1-\omega(n T) d_{1}\right) \eta\left(e^{r T}-1\right) P(n T)}$.

Denote $Y_{n}=P(n T)$, then we have the following non-autonomous difference equation

$Y_{n+1}=\frac{\left(1-\omega(n T) d_{1}\right) e^{r T} Y_{n}}{1+\left(1-\omega(n T) d_{1}\right) \eta\left(e^{r T}-1\right) Y_{n}}$,

this is similar to the well-known Beverton-Holt model [18-20]. Note that the difference Eq. (14) is very dynamic since $\omega(n T)$ depends on the third equation of model (11), which indicates that the difference equation discussed in present work is much more difficult than those in references [21-23]. In fact, the non-autonomous or impulsive Beverton-Holt difference equations have been studied extensively. For example, the asymptotic properties of BevertonHolt difference equation have been discussed by Berezansky and Braverman [21] and Kocic [22,23]. In this work, based on our practical problem we are interested in the stability of zero solution of Eq. (14). It is seen that the inequality

$Y_{n+1}<\left(1-\omega(n T) d_{1}\right) e^{r T} Y_{n}$

holds true for all $n \in \mathcal{N}$. So we can define the dynamic threshold value $R_{0}(n, T)$ as follows

$R_{0}(n, T) \doteq\left(1-d_{1} \omega(n T)\right) e^{r T}$.

with $\omega(n T))$ is given by (9). Therefore, if $R_{0}(n, T)<1$ for all $n \in \mathcal{N}$, then the zero solution of Eq. (14), i.e. non-autonomous difference Eq. (14), is globally asymptotically stable.

This indicates that the pest population will die out if the threshold value $R_{0}(n, T)<1$ for all $n \in \mathcal{N}$. The threshold value is very dynamic and depends on the number of pesticide applications, and we will show this in more detail later.
In particular, if $q_{n}=1$ for $n \in \mathcal{N}$ (i.e. $\omega(t)$ satisfies Eq. (3)), then

$R_{0}(n, T)=\left(1-\frac{d_{1} \omega_{0}}{\omega_{0}+\left(1-\omega_{0}\right) e^{d_{1} n T}}\right) e^{r T} \doteq R_{0}^{1}(n, T)$.

Note that, in reality, $R_{0}(n, T)<1$ may hold true for the first few pesticide applications and then it will increase and exceed 1 due to the evolution of pest resistance. Fig. 2 provides an example to show how the density of the pest population and the threshold value $R_{0}(n, T)$ change as the number of pesticide applications increases. If we fixed all parameter values as those in Fig. 2 and let period $T$ vary, Fig. 2(a) gives three numerical solutions with different period $T$. Here we stop to integrate Eq. (11) until the density of the pest population exceeds a given threshold value $T H$ (such as EIL). It is seen that the density of the pest population at time points $n T$ decreases firstly due to the high efficacy of the pesticides, and then increases because of the evolution of pest resistance. For more details, see Fig. 3. The threshold value $R_{0}(n, T)$ corresponding to three solutions in Fig. 2(a) at each time point $n T$ is given in Fig. 2(b).

It is interesting to note that resurgence by the pest population can occur very quickly once the pest resistance develops. Fig. 2(a) also shows that the less frequent (larger period $T$ ) are the pesticide applications, the higher the level that pest outbreaks can reach. However, as mentioned before, the higher the frequency of pesticide applications, the faster the evolution of pest resistance. Therefore, the question is how to manage the pest resistance, i.e. what is the optimal time to switch to a new type of pesticide? We address this question in the following subsection.

\subsection{Justifications and the optimal time to switch pesticides}

As mentioned in the introduction, the density of resistant pests will grow quickly if one kind of pesticide is sprayed frequently (as shown in Fig. 2(a)), and it will lead to a pest outbreak or 
(a)

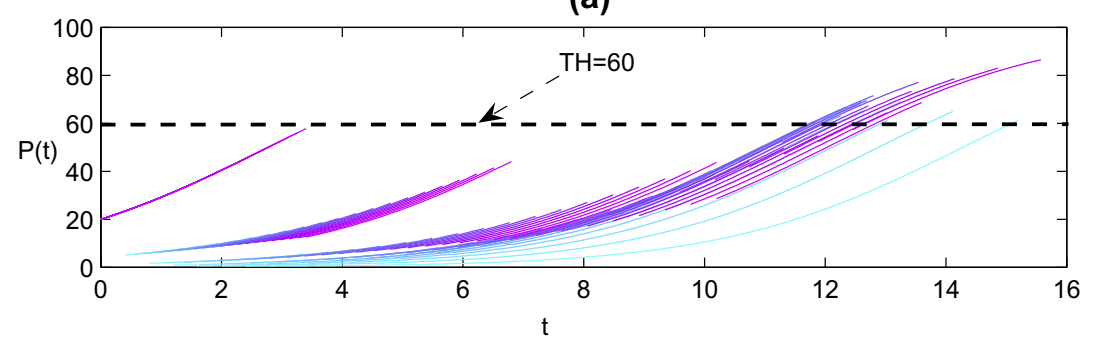

(b)

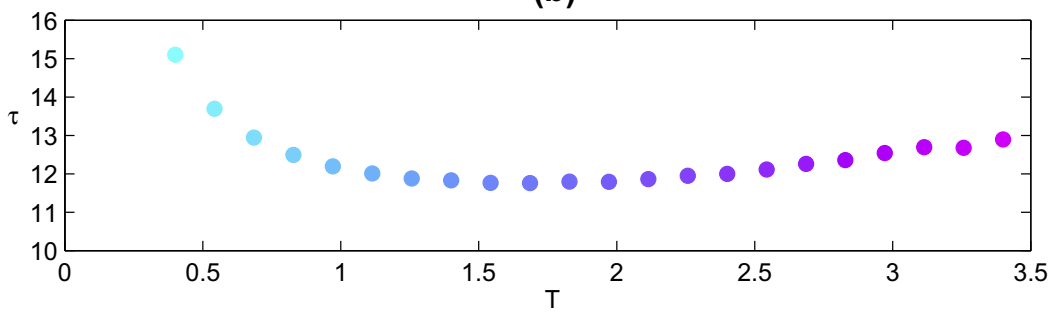

(c)

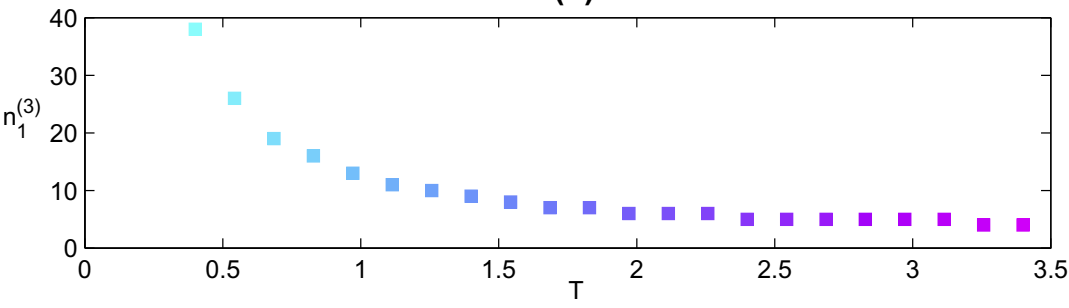

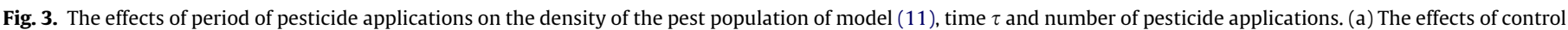

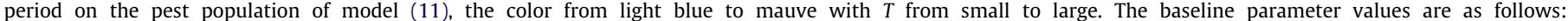

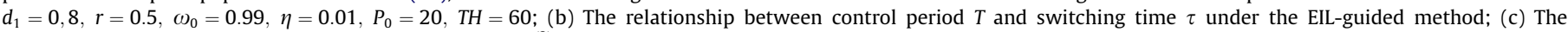

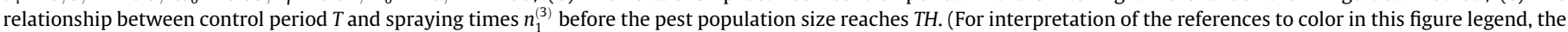
reader is referred to the web version of this article.)

resurgence. Therefore, people usually switch pesticides at some given time and how to choose the optimal time for a switch is an important practical question. In the following, we will provide three different methods based on our model (11) to choose the optimal time for switching pesticides according to different justifications. We should emphasize that for each new type of pesticide, we assume that the evolution of pesticide resistance, i.e. $\omega$, follows the same equation and has the same initial condition $\omega_{0}$.

Method 1 Optimal time for switching pesticides with $R_{0}(n, T)$ as a guide.

It follows from Fig. 2(b) that $R_{0}(n, T)$ is increasing with respect to $n$. Therefore, in order to eradicate the pest population we should maintain $R_{0}(n, T)<1$ to hold true for all $n \in \mathcal{N}$. This clarifies that we need to switch the pesticide once the threshold value $R_{0}(n, T)$ goes to one. Without loss of generality, we assume that the threshold value $R_{0}(n, T)$ will exceed one unit after $n_{1}^{(1)}$ pesticide applications, i.e.

$n_{1}^{(1)}=\max \left\{n: R_{0}(n, T) \leqslant 1\right\}$.

In order to find $n_{1}^{(1)}$, we let $R_{0}(n, T)=1$, then

$\omega(n T)=\frac{1-e^{-r T}}{d_{1}}$,

where $\omega(n T)$ is given by (9). Therefore,

$n_{1}^{(1)}=\left[\left\{n: \omega(n T)=\frac{1-e^{r T}}{d_{1}}\right\}\right]$, and $[a]$ denotes the greatest integer no larger than $a$.

In particular, if $q_{n}=1$ then $R_{0}(n, T)=R_{0}^{1}(n, T)$. Let $R_{0}^{1}(n, T)=1$, we can solve the above equation with respect to $n$ and yield the optimal switching time $n_{1}^{(1)} T$, where

$n_{1}^{(1)}=\left[\frac{1}{d_{1} T} \ln \frac{\omega_{0}-\left(1-d_{1}\right) \omega_{0} e^{r T}}{\left(1-\omega_{0}\right)\left(e^{r T}-1\right)}\right]$

If we switch pesticides according to this decision strategy, i.e. a threshold condition-guided method, then the pest population can be completely eradicated after several pesticide switches. To show this, Fig. 4(a) depicts numerical simulation under this strategy. It follows from Fig. 4(a) that the pest population will die out eventually, where $n_{1}^{(1)}=2$. That is after three pesticide applications of one kind of pesticide (note that the first pesticide application is at time $t=0$ ), the farmers must switch to another kind of pesticide to eradicate the pest quickly.

Method 2 Optimal time for switching pesticides with $P(n T)$ as a guide.

Note that $\omega(n T) d_{1}$ represents the instant killing rate of the pesticide at time $n T$, and $\Delta P(n T)=P(n T)-P\left(n T^{+}\right)=\omega(n T) d_{1} P(n T)$ denotes the number of pests killed after spraying pesticide at time $n T$. It follows from Fig. 2(a) and the evolution of $\omega$ that if the spraying period is fixed, $\{\Delta P(n T)\}$ is a monotonically decreasing sequence, and $\Delta P(n T) \rightarrow 0$ as $n \rightarrow \infty$ due to $\omega(n T) \rightarrow 0$. However, $P(n T)$ is not monotonic, which indicates that the effects of the pesticide wear off naturally with increasing spraying times because of the increasing pest resistance. 
(a)

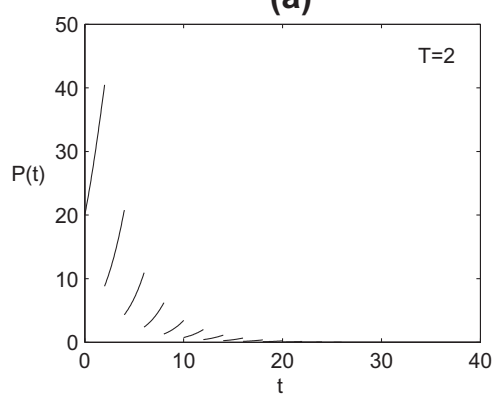

(c)

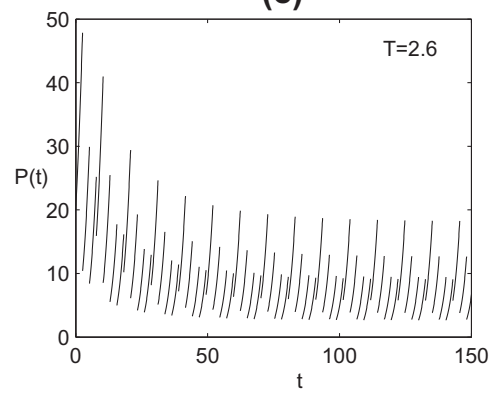

(b)

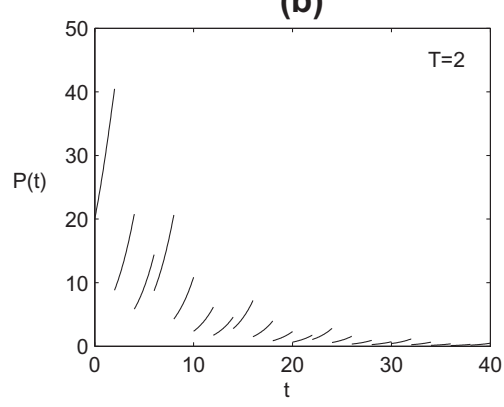

(d)

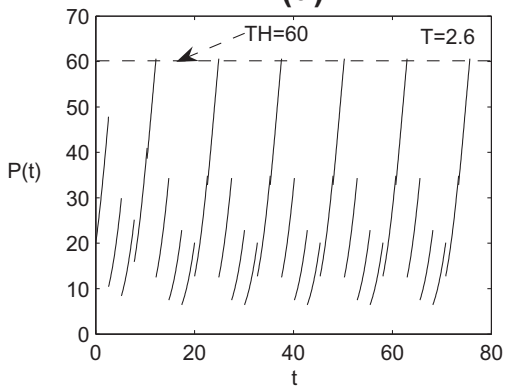

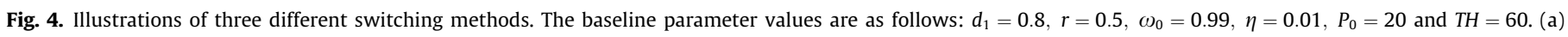

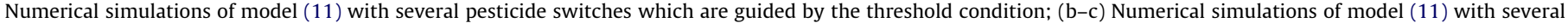

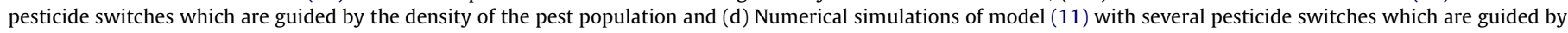
the EIL.

Based on the observations from Figs.2(a) and 3(a), we denote the sequence $\{P(n T)\}_{n \in \mathcal{N}}$ as the density of the pest population at time points $n T$, and for each fixed period $T$, there exists an integer $n_{1} \in \mathcal{N}$ such that

$P(T)>P(2 T)>\cdots>P\left(\left(n_{1}-1\right) T\right)$

and

$P\left(\left(n_{1}-1\right) T\right)<P\left(n_{1} T\right)<P\left(\left(n_{1}+1\right) T\right)<\cdots$,

where the sequence $\{P(n T)\}_{n \in \mathcal{N}}$ satisfies the following iteration equation

$$
\begin{aligned}
P(n T) & =\frac{\left(1-\omega((n-1) T) d_{1}\right) P((n-1) T) e^{r T}}{1+\left(1-\omega((n-1) T) d_{1}\right) P((n-1) T) \eta\left(e^{r T}-1\right)} \\
& =\frac{P_{0} e^{n r T} \prod_{j=1}^{n-1}\left(1-\omega(j T) d_{1}\right)}{1+P_{0} \eta\left(e^{r T}-1\right)\left(1+\sum_{j=1}^{n-1} e^{j r T}\left(\prod_{k=1}^{j}\left(1-\omega(k T) d_{1}\right)\right)\right)} .
\end{aligned}
$$

Since the efficacy of the pesticides wears off with increasing spraying, the pesticide should be changed once the efficacy of the pesticide decreases to the level at which it cannot kill enough pests and $\{P(n T)\}$ begins to increase. That is, farmers or other pest managers should switch pesticide when the pest density first satisfies the condition $P(n T)>P((n-1) T)$, as shown in Figs.2(a) and $3(\mathrm{a})$. Therefore, the optimal time for switching pesticide is $n_{1}^{(2)} T$, where $P\left(\left(n_{1}^{(2)}-1\right) T\right)$ is the minimum value of sequence $\{P(n T)\}_{n \in \mathcal{N}}$.

In order to show how to determine $n_{1}^{(2)}$, we denote

$f^{(n)}(x)=\frac{\left(1-\omega(n T) d_{1}\right) e^{r T} x}{1+\left(1-\omega(n T) d_{1}\right) \eta\left(e^{r T}-1\right) x} \doteq \frac{a(n) x}{1+b(n) x}$,

where $a(n)=\left(1-\omega(n T) d_{1}\right) \exp (r T)=R_{0}(n, T), \quad b(n)=(1-\omega(n T)$ $\left.d_{1}\right) \eta(\exp (r T)-1)$. Then for fixed $n$, there is an autonomous difference equation determined by function $f^{(n)}$, i.e. we have
$P(n T)=f^{(n)}(P(n-1) T)=\frac{A P((n-1) T)}{1+B P((n-1) T)}, n \in \mathcal{N}$

with $A=a(n), B=b(n)$. For example, if we only apply the pesticide $N_{1}$ times, then we have

$$
\begin{aligned}
P(T) & =f^{(1)}(P(0)), P(2 T)=f^{(2)}(P(T)), \ldots, P\left(N_{1} T\right) \\
& =f^{\left(N_{1}-1\right)}\left(P\left(\left(N_{1}-1\right) T\right)\right) .
\end{aligned}
$$

Therefore, for a given $P(0)$, the time series $P(T), P(2 T), \ldots$ can be obtained. In order to investigate how the period $T$ and the $q_{n}$ function affect the time of pesticide switching, i.e. the value $n_{1}^{(2)} T$, we carry out dynamic cobweb analysis, as shown in Fig. 5. To do this, we fixed all parameter values as those in Fig. 5 and chose different period $T$ and $q_{n}$ functions for each subplot. Here we assume $N_{1}=10$, i.e. the same pesticide is applied 10 times periodically, and the development of the sequence $\{P(n T)\}_{n \in \mathcal{N}}$ with an initial value $P(0)=90$ for given period $T$ and $q_{n}$ function are given in Fig. 5 . The colors from light blue to mauve with $n$ from 1 to 10 provide the iteration function $f^{(n)}, n=1,2, \ldots, 10$. It is clear that the sequence $\{P(n T)\}_{n \in \mathcal{N}}$ reaches its minimum value and then increases. This also clarifies the number of pesticide applications (i.e. $n_{1}^{(2)}+1$ ) before the pest can be resurgent for a given initial population size $(P(0)=90$ in all simulations) and how fast the pest can reach its carrying capacity (here $(a(n)-1) / b(n)$ in Fig. 5 , see more details in the following discussion). All these results confirm that the value $n_{1}^{(2)}$ depends on the period $T$ and function $q_{n}$.

In order to get an analytical expression of $n_{1}^{(2)}$ for this decision strategy, we employ results on the well-known Beverton-Holt model again. For any fixed $n$, we have the following Beverton-Holt difference equation

$x_{k}=f^{(n)}\left(x_{k-1}\right)=\frac{A x_{k-1}}{1+B x_{k-1}}, \quad k \in \mathcal{N}$ 

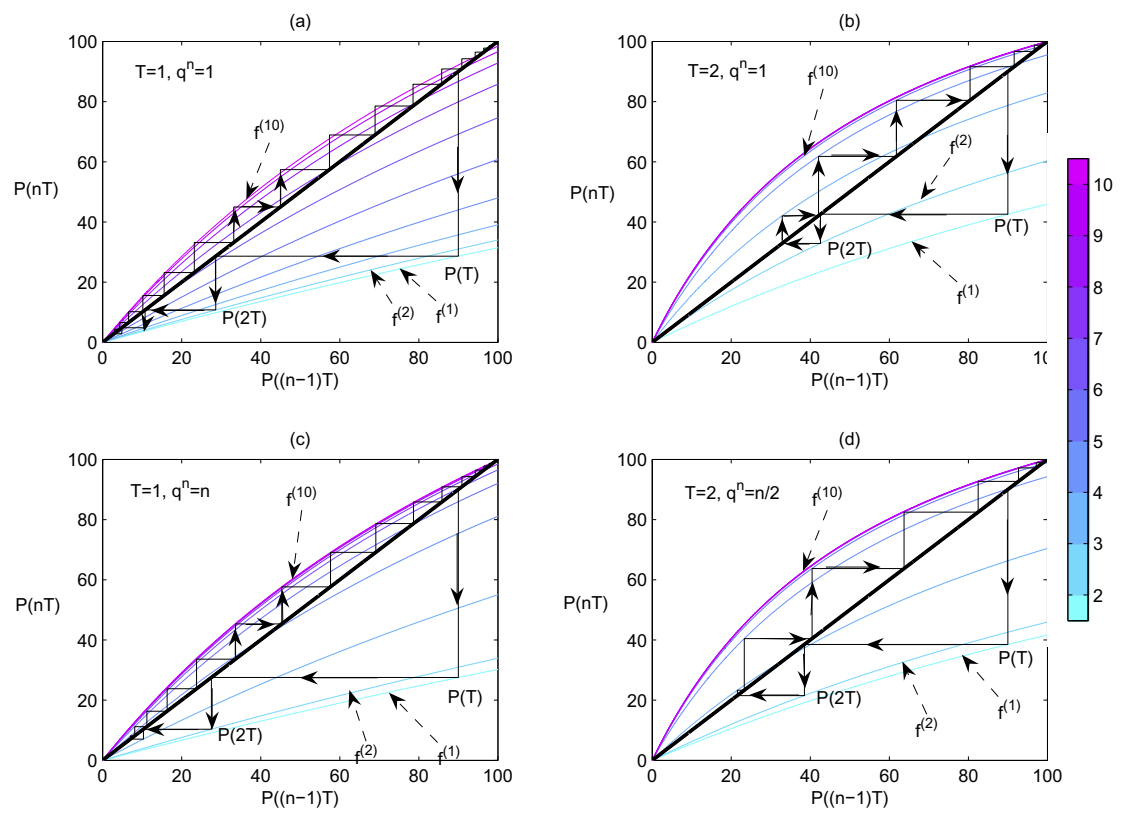

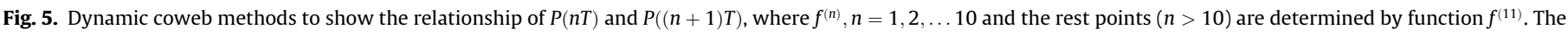

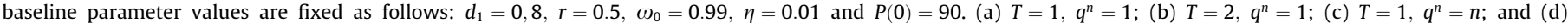
$T=2, q^{n}=n / 2$

which has two steady states, $x_{1}^{*}=0$ (it is stable if $A<1$ ) and $x_{2}^{*}=(A-1) / B$ (it is stable if $A \geqslant 1$ ), and $x_{k}>x_{k-1}$ holds true if $x_{k-1}<(A-1) / B$ and $A>1$.

Based on this fact and the relationship of $P((n-1) T)$ and $P(n T)$, we have

$P(n T)>P((n-1) T) \Longleftrightarrow P((n-1) T)<\frac{a(n-1)-1}{b(n-1)}$

with $a(n-1)=R_{0}(n-1, T)>1$.

Therefore,

$n_{1}^{(2)}=\left[\left\{n: P((n-1) T)=\frac{a(n-1)-1}{b(n-1)}, R_{0}(n-1, T)>1\right\}\right]$.

It follows from (17) and (21) that we have $n_{1}^{(2)}>n_{1}^{(1)}$. This implies that the switching time with the threshold condition-guided method is less than the switching time with the pest population density-guided method.

Fig. 4(b) and (c) give numerical simulations with pesticide switching tactics. Fig. 4(b) shows that the pest population dies out eventually and $n_{1}^{(2)}=4$. However, if the pesticide application period $T$ is larger than some critical values, the pest population will oscillate periodically under this pesticide switching strategy (see Fig. 4(c)). This indicates that if the pesticide application period $T$ is too large, the pest population may have an outbreak and exceed the EIL. If so, we need to combine the method with other feasible decision switching options in order to determine the pesticide application period properly.

Method 3 Optimal time for switching pesticides with EIL as a guide.

Farmers and other pest managers usually control pests such that they cannot exceed the EIL. From Fig. 2(a) we can see that the pest population grows very quickly and even exceeds the EIL (denoted by $\mathrm{TH}$ ) after repeated spraying with one type of pesticide, because of the accumulation of the pest's resistance to it. Therefore, what we want to address is if the switch action is only initiated when the pest population's size reaches a given level $T H$, how can the time when the pest population reaches $T H$ be determined and how frequently do we need to switch the pesticides?
Without loss of generality, assume that the component of $P(t)$ of any solution of model (11) with initial value $P_{0}$ will reach $\mathrm{TH}$ at time $\tau$, then from (12) we get

$T H=\frac{\left(1-\omega\left(n_{1}^{(3)} T\right) d_{1}\right) P\left(n_{1}^{(3)} T\right) e^{r\left(\tau-n_{1}^{(3)} T\right)}}{1+\left(1-\omega\left(n_{1}^{(3)} T\right) d_{1}\right) P\left(n_{1}^{(3)} T\right) \eta\left(e^{r\left(\tau-n_{1}^{(3)} T\right)}-1\right)}$,

where $n_{1}^{(3)}>0$ is the minimum spraying number such that the density of the pest population $P\left(\left(n_{1}^{(3)}+1\right) T\right)$ exceeds $T H$ firstly, and $n_{1}^{(3)}$ can be calculated from Eq. (18). Solving the above equation with respect to $\tau$ yields

$\tau=\frac{1}{r} \ln \frac{T H\left(1-\left(1-\omega\left(n_{1}^{(3)} T\right) d_{1}\right) P\left(n_{1}^{(3)} T\right) \eta\right)}{\left(1-\omega\left(n_{1}^{(3)} T\right) d_{1}\right) P\left(n_{1}^{(3)} T\right)(1-\eta T H)}+n_{1}^{(3)} T$.

Fig. 3 provides an example to show how the different spraying periods affect the density of the pest population, the time $\tau$ and spraying number $n_{1}^{(3)}$. Fig. $4(\mathrm{~d})$ gives the numerical simulation under this tactic of switching pesticides, from which we can see that the control action will tend to periodic control after a certain number of times that the pesticides are switched, where $n_{1}^{(3)}=5$. It follows from Fig. 3(b) that the time $\tau$ is not a monotonic function of the spraying period, while the minimum number of pesticide applications $n_{1}^{(3)}$ is decreasing as $T$ increases (see Fig. 3(c)). In practice, one of the main purposes of pest resistance management is to delay the evolution of resistance under condition of eradication of the pest population or maintaining its density below EIL. Our results presented in Fig. 3 show that the relationship between $\tau$ and $T$ is complex. Thus, how to choose the spraying times and how to manage pest resistance such that the pest control is costeffective is a challenge.

\section{Comparison of the three methods for deciding the optimal time to switch pesticides}

From the above discussion, we know that the pest population dies out under the threshold value-guided method, and it follows from Fig. 4(b) and (c) that the pest population either dies out or 
tends to a periodic solution under the $P(n T)$ guided method, depending on the period of pesticide applications, while the pest population oscillates with a maximum value $T H$ under the EIL guided method. The question is which method is an optimal choice in practice? We discuss this question below.

We first would like to derive the conditions to determine if the pest population dies out or oscillates periodically under the density of pest population $P(n T)$ guided method.

Let $n_{i}$ be the number of effective pesticide applications of the $i$ th pesticide switches and $P^{(i)}(k T)$ is the pest population at time $k T$ after the $i$ th pesticide switch, that is $P^{(i)}(k T)=P\left(\left(\sum_{j=1}^{i} n_{j}+k\right) T\right)$ and $P^{(i)}(0)=P^{(i-1)}\left(n_{i-1} T^{+}\right)=\left(1-\omega\left(n_{i-1} T\right) d_{1}\right) P^{(i-1)}\left(n_{i-1} T\right)$. According to Eq. (18) we have

$P^{(i)}\left(n_{i} T\right)=\frac{\left(1-\omega\left(n_{i-1} T\right) d_{1}\right) P^{(i-1)}\left(n_{i-1} T\right) e^{n_{i} T T} \prod_{j=1}^{n_{i}-1}\left(1-\omega(j T) d_{1}\right)}{1+\left(1-\omega\left(n_{i-1} T\right) d_{1}\right) P^{(i-1)}\left(n_{i-1} T\right) \eta\left(e^{r T}-1\right)\left(1+\sum_{j=1}^{n_{i}-1} e^{j r T}\left(\prod_{k=1}^{j}\left(1-\omega(k T) d_{1}\right)\right)\right)}$.

Denote $Y^{(i)}=P^{(i)}\left(n_{i} T\right)$, then we have the following difference equation

$Y^{(i)}=\frac{\left(1-\omega\left(n_{i-1} T\right) d_{1}\right) Y^{(i-1)} e^{n_{i} T} \prod_{j=1}^{n_{i}-1}\left(1-\omega(j T) d_{1}\right)}{1+\left(1-\omega\left(n_{i-1} T\right) d_{1}\right) Y^{(i-1)} \eta\left(e^{r T}-1\right)\left(1+\sum_{j=1}^{n_{i}-1} e^{j r T}\left(\prod_{k=1}^{j}\left(1-\omega(k T) d_{1}\right)\right)\right)}$.

Again this is the well-known Beverton-Holt model, which has a zero equilibrium $Y_{1}^{*}=0$. It is stable provided that

$g(T) \doteq\left(1-\omega\left(n_{i-1} T\right) d_{1}\right) e^{n_{i} r T} \prod_{j=1}^{n_{i}-1}\left(1-\omega(j T) d_{1}\right)<1$.

If $g(T)>1$, then (23) has a stable positive equilibrium

$Y_{2}^{*}=\frac{\left(1-\omega\left(n_{i-1} T\right) d_{1}\right) e^{n_{i} r T} \prod_{j=1}^{n_{i}-1}\left(1-\omega(j T) d_{1}\right)-1}{\left(1-\omega\left(n_{i-1} T\right) d_{1}\right) \eta\left(e^{r T}-1\right)\left(1+\sum_{j=1}^{n_{i}-1} e^{j r T}\left(\prod_{k=1}^{j}\left(1-\omega(k T) d_{1}\right)\right)\right)}$.

Let $T_{1}$ be the solution of equation $g(T)=1$, then if $T<T_{1}$, the pest population will die out after several pesticide switchings, and if $T>T_{1}$, the pest population will fluctuate periodically.

Therefore, if we aim to eradicate the pest population and the period of pesticide applications must satisfy $T<T_{1}$, our results support the density guided method (i.e. method 2 ) because of $n_{1}^{(2)}>n_{1}^{(1)}$, as shown in Fig. 4(b).

If the period of pesticide applications $T$ is larger than $T_{1}$, then the periodic switching of pesticides will result in oscillating of the pest population periodically under the density guided method, as shown in Fig. 4(c). However, for this case the amplitude of the pest population could be very large and its maximum value can exceed EIL (i.e. $Y^{*}>T H$ ), which will result in great economic loss. These results confirm that if $T \geqslant T_{1}$, the tactic of switching pesticides with the EIL-guided method is better than the tactic of switching pesticides with the density guided method, because the EIL-guided method is in good agreement with the aims of an IPM strategy, that is control action must be taken once a critical density of the pests is observed in the field so that the EIL is not exceeded $[6,8-10]$.

\section{Discussion}

Chemical methods in IPM are the most direct and effective [6,8-10]. However, frequent use of one kind of pesticide in the long-term may create selection pressure for evolution of pest resistance to the pesticide. If too large a proportion of a pest population develops resistance to the pesticide toxin, the susceptibility of the entire pest population to the pesticide toxin will be lost eventually, leading to pest resurgences and outbreaks.
Natural enemies may keep a pest population relatively stable in the absence of any pesticide application. Indeed, a key component of an IPM strategy is often biological control [24,25], which is defined as the reduction of pest populations by natural enemies. It typically involves impulsive perturbations, such as the release of natural enemies at a critical time of the season when insufficient reproduction of the natural enemies already present is likely to occur and pest control will be achieved exclusively by the released individuals themselves (augmentation) [26,27].

Pulse-like pest management actions such as spraying pesticides and killing a pest instantly and the release of natural enemies at critical times can be modelled with impulsive differential equations. Recently, many mathematical models with impulsive chemical control tactics and releases of natural enemies have been proposed to model an IPM strategy such as spraying of pesticides [25,28-33] or releases of natural enemies at critical times [27,34-41]. Those studies mainly focused on the effects of chemical control and biological control on the permanence or extinction of pest populations, and did not consider the effects of pesticide resistance. In particular, they paid no attention to questions such as (1) how to determine the period of pesticide switches? (2) What is the optimal justification for switching from one kind pesticide to another, unrelated, new kind pesticide? And (3) how to determine the time when the density of the pest population reaches or exceeds EIL? All these questions are key issues for the management of pesticide resistance and applying cost-effective pest control strategies in practice.

To answer such questions, we developed a pest population growth model including pesticide resistance. In particular, we investigated how the number of pesticide applications or the frequency of pesticide applications affects the evolution of pesticide resistance, and consequently affects the success or failure of pest control.

In particular, we have provided three possible methods which can help us to judge when we should switch pesticides: the threshold condition-guided method; the density of pest population-guided method and the EIL-guided method. If we want to completely eradicate the pest population, then the threshold condition-guided method or density-guided method can be employed to determine the frequency of pesticide switches. However, our results confirm that if we properly choose the period of pesticide application, the density-guided method is better than the threshold condition-guided method in terms of the timing for pesticide switching. Moreover, the method for determining the period of pesticide applications is also provided. We also would like to point out that this guided method may be easily implemented in practice, because we only need to record the density of the pest population at the time of each pulse spraying of pesticides and monitor the density at the most recent time of pulse spraying. But we should emphasize here that the period of pesticide applications must be carefully designed based on our method.

If we want to maintain the density of pests below some predefined threshold value such as EIL rather than eradicating the pest population, then the density-guided method or EIL-guided method can be employed to determine the frequency of pesticide switches. Our results confirm that the EIL-guided method should also be used to determine the timing of pesticide applications, due to the uncertainties involved in the density-guided method, i.e. the amplitude of the pest population may be very large (vast pest outbreaks may occur, e.g. in locust populations) if the period of pesticide applications is not chosen properly. Moreover, the EILguided method can maintain the density of pest populations below the EIL forever, which is one of the main purposes of IPM strategies $[6,8-10,42]$. Note that the time when the pest population grows and reaches the EIL is not monotonic with respect to the frequency of the pesticide applications. This indicates that although the 
effects of the pesticide naturally wear off with the increasing of spraying times, the effects of the period of pesticide applications on their timing $\tau$ shown in Fig. 3 is complex.

To avoid multiple resistance, we need to adopt the IPM approach [7]. Therefore, we will extend our single pest population growth model to include natural enemies in the future. Due to the pesticide wearing off, repeated releases of the same number of natural enemies is either insufficient as they no longer suppress the pest population once the pest resistance develops, or the number released is too large which is not cost effective and may cause secondary outbreaks or pest resurgence. If so, how should one determine the new number of natural enemies to be released at each control action? This question will be addressed in future research. Meanwhile, the growth rate of a pest population can be strongly impacted by environmental conditions, thus the pest control strategies should be adaptable to changing conditions. Therefore, both stochasticity in growth rate and the ability to adapt control methods in response to changing conditions will be also addressed to make the model more realistic and practical.

\section{Acknowledgments}

This work was partially supported by the National Natural Science Foundation of China (11171199), and by the Fundamental Research Funds for the Central Universities (GK201104009, GK201302004).

\section{References}

[1] M.B. Thomas, Ecological approaches and the development of truly integrated pest management, Proc. Nat. Acad. Sci. 96 (1999) 5944.

[2] G.P. Georghiou, Overview of Insecticide Resistance, in: M.B. Green, H.M. LeBaron, W.K. Moberg (Eds.), Managing Resistance to Agrochemicals: From Fundamental Research to Practical Strategies, American Chemical Society, Washington DC, 1990 , p. 18.

[3] M.J. Kotchen, Incorporating Resistance in Pesticide Management: A Dynamic Regional Approach, Springer Verlag, New York, 1999 (p. 126).

[4] Pesticides 101 - A Primer, http://<www.panna.org/issues/pesticides-101primer>.

[5] Y. Dumont, J.M. Tchuenche, Mathematical studies on the sterile insect technique for the Chikungunya disease and Aedes albopictus, J. Math. Biol. (2011), http://dx.doi.org/10.1007/s00285-011-0477-6 (in print).

[6] M.L. Flint, Integrated Pest Management for Walnuts, University of California Statewide Integrated Pest Management Project, Division of Agriculture and Natural Resources, second ed., Oakland, CA, Publication, University of California, 1987 (p. 3270)

[7] D.W. Onstad, Insect Resistance Management, Elsevier, Amsterdam, 2008

[8] J.C. Van Lenteren, J. Woets, Biological and integrated pest control in greenhouses, Annu. Rev. Entomol. 33 (1988) 239.

[9] J.C. Van Lenteren, Integrated Pest Management in Protected Crops, in: D. Dent (Ed.), Integrated Pest Management, Chapman \& Hall, London, 1995, p. 311.

[10] J.C. Van Lenteren, Measures of Success in Biological Control of Arthropods By Augmentation of Natural Enemies, in: S. Wratten, G. Gurr (Eds.), Measures of Success in Biological Control, Kluwer Academic Publishers, Dordrecht, 2000, p. 77.

[11] D. Kurtak, R. Meyer, M. Ocran, M. Ouedraogo, P. Renaud, R.O. Sawadogo, B. Tele, Management of insecticide resistance in control of the Simulium damnosum complex by the Onchocerciasis Control programme, West Africa: potential use of negative correlation between organophosphate resistance and pyrethroid susceptibility, Med. Veterinary Entomol. 1 (1987) 137.

[12] P.J. Cameron, G.P. Walker, Diamondback Moth Resistance Management and Prevention Strategy, in: N.A. Martin, R.M. Beresford, K.C. Harrington (Eds.), Pesticide Resistance: Prevention and Management Strategies 2005, The New Zealand Plant Protection Society, Hastings, New Zealand, 2005, p. 49.

[13] R.J. Hall, S. Gubbins, C.A. Gilligan, Invasion of drug and pesticide resistance is determined by a trade-off between treatment efficacy and relative fitness, Bull. Math. Biol. 66 (2004) 825.
[14] S. Bonhoeffer, M.A. Nowak, Pre-existence and emergence of drug resistance in HIV-1 infection, Proc. R. Soc. London B 264 (1997) 631.

[15] S. Gubbins, C.A. Gilligan, Invasion thresholds for fungicide resistance: deterministic and stochastic analyses, Proc. R. Soc. London B 266 (1999) 2539.

[16] R. Laxminarayan, R.D. Simpson, Biological limits on agricultural intensification: an example from resistance management, Resour. Future (2000) 47.

[17] M.G. Milgroom, S.A. Levin, W.E. Fry, Population Genetics Theory and Fungicide Resistance, in: K.J. Leonard, W.E. Fry (Eds.), Plant Disease Epidemiology 2: Genetics, Resistance and Management, McGraw-Hill, New York, 1989, p. 340.

[18] R.J.H. Beverton, S.J. Holt, On the Dynamics of Exploited Fish Populations, Fishery Investigations, Series 2, Vol. 19, H.M. Stationery Office, London, 1957.

[19] $A^{\circ}$ Brännström, D.J.T. Sumpter, The role of competition and clustering in population dynamics, Proc. R. Soc. B 272 (2005) 2065.

[20] S.A.H. Geritz, Éva Kisdi, On the mechanistic underpinning of discrete-time population models with complex dynamics, J. Theor. Biol. 228 (2004) 261.

[21] L. Berezansky, E. Braverman, On impulsive Beverton-Holt difference equation and their applications, J. Differ. Equ. Appl. 10 (2004) 851.

[22] V.L. Kocic, D. Stutson, G. Arora, Global behavior of solutions of a nonautonomous delay logistic difference equation, J. Differ. Equ. Appl. 10 (2004) 1267.

[23] V.L. Kocic, A note on the nonautonomous Beverton-Holt model, J. Differ. Equ. Appl. 11 (2005) 415.

[24] D.J. Greathead, Natural Enemies of Tropical Locusts and Grasshoppers: Their Impact and Potential as Biological Control Agents, in: C.J. Lomer, C. Prior (Eds.), Biological Control of Locusts and Grasshoppers, C.A.B. International, Wallingford, UK, 1992, p. 105.

[25] F.D. Parker, Management of Pest Populations by Manipulating Densities of Both Host and Parasites Through Periodic Releases, in: C.B. Huffaker (Ed.), Biological Control, Plenum Press, New York, 1971.

[26] M.P. Hoffmann, A.C. Frodsham, Natural Enemies of Vegetable Insect Pests, Cooperative Extension, Cornell University, Ithaca, NY, 1993 (p. 63).

[27] P. Neuenschwander, H.R. Herren, Biological control of the Cassava Mealybug, Phenacoccus manihoti, by the exotic parasitoid Epidinocarsis lopezi in Africa, Phil. Trans. R. Soc. London B 318 (1988) 319.

[28] C.T. Li, S.Y. Tang, The dynamical behavior of non-smooth system with impulsive control strategies, Int. J. Biomath. 5 (1) (2012), http://dx.doi.org/ 10.1142/S1793524511001532.

[29] J.H. Liang, S.Y. Tang, Optimal dosage and economic threshold of multiple pesticide applications for pest control, Math. Comput. Model. 51 (2010) 487.

[30] Y.S. Tan, J.H. Liang, S.Y. Tang, The effects of timing of pulse spraying and releasing periods on dynamics of generalized predator-prey model, Int. J. Biomath. 5 (3) (2012), http://dx.doi.org/10.1142/S1793524512600182.

[31] L.M. Wang, L.S. Chen, J.J. Nieto, The dynamics of an epidemic model for pest control with impulsive effect, Nonlinear Anal.: Real World Appl. 11 (2010) 1374.

[32] Y. Xue, S.Y. Tang, J.H. Liang, Optimal timing of interventions in fishery resource and pest management, Nonlinear Anal.: Real World Appl. 13 (2012) 1630.

[33] H. Zhang, L.S. Chen, J.J. Nieto, A delayed epidemic model with stage-structure and pulses for pest management strategy, Nonlinear Anal.: Real World Appl. 9 (2008) 1714.

[34] J.J. Jiao, S.H. Cai, L.S. Chen, Analysis of a stage-structured predator-prey system with birth pulse and impulsive harvesting at different moments, Nonlinear Anal: Real World Appl. 12 (2011) 2232.

[35] J.H. Liang, S.Y. Tang, An integrated pest management model with delayed responses to pesticide applications and its threshold dynamics, Nonlinear Anal.: Real World Appl. 13 (2012) 2352.

[36] S.Y. Tang, Y.N. Xiao, L.S. Chen, R.A. Cheke, Integrated pest management models and their dynamical behaviour, Bull. Math. Biol. 67 (2005) 115.

[37] S.Y. Tang, Y.N. Xiao, R.A. Cheke, Multiple attractors of host-parasitoid models with integrated pest management strategies: eradication, persistence and outbreak, Theor. Popul. Biol. 73 (2008) 181

[38] S.Y. Tang, R.A. Cheke, Models for integrated pest control and their biological implications, Math. Biosci. 215 (2008) 115.

[39] S.Y. Tang, Y.N. Xiao, R.A. Cheke, Effects of predator and prey dispersal on success or failure of biological control, Bull. Math. Biol. 71 (2009) 2025.

[40] S.Y. Tang, G.Y. Tang, R.A. Cheke, Optimum timing for integrated pest management: modelling rates of pesticide application and natural enemy releases, J. Theor. Biol. 264 (2010) 623.

[41] S.Y. Tang, J.H. Liang, Y.S. Tan, R.A. Cheke, Threshold conditions for integrated pest management models with pesticides that have residual effects, J. Math. Biol. 66 (2013) 1.

[42] W. Zhang, S.M. Swinton, Optimal control of soybean aphid in the presence of natural enemies and the implied value of their ecosystem services, J. Environ. Manage. 96 (2012) 7. 